\title{
Geomorphology of the Imhotep region on comet 67P/Churyumov-Gerasimenko from OSIRIS observations
}

\author{
A.-T. Auger ${ }^{1,2}$, O. Groussin ${ }^{1}$, L. Jorda ${ }^{1}$, S. Bouley ${ }^{2,3}$, R. Gaskell ${ }^{4}$, P. L. Lamy ${ }^{1}$, C. Capanna ${ }^{1}$, N. Thomas ${ }^{5}$,

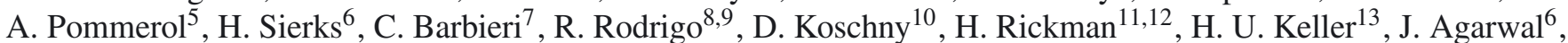 \\ M. F. A'Hearn ${ }^{14}$, M. A. Barucci ${ }^{15}$, J.-L. Bertaux ${ }^{16}$, I. Bertini ${ }^{17}$, G. Cremonese ${ }^{18}$, V. Da Deppo ${ }^{19}$, B. Davidsson ${ }^{11}$, \\ S. Debei ${ }^{18}$, M. De Cecco ${ }^{20}$, M. R. El-Maarry ${ }^{5}$, S. Fornasier ${ }^{15}$, M. Fulle ${ }^{21}$, P. J. Gutiérrez ${ }^{22}$, C. Güttler ${ }^{6}$, S. Hviid ${ }^{23}$, \\ W.-H. Ip ${ }^{24}$, J. Knollenberg ${ }^{23}$, J.-R. Kramm ${ }^{6}$, E. Kührt ${ }^{23}$, M. Küppers ${ }^{25}$, F. La Forgia ${ }^{7}$, L. M. Lara ${ }^{22}$, M. Lazzarin ${ }^{7}$, \\ J. J. Lopez Moreno ${ }^{22}$, S. Marchi ${ }^{26}$, F. Marzari ${ }^{7}$, M. Massironi' ${ }^{27,28}$, H. Michalik ${ }^{29}$, G. Naletto ${ }^{30,17,19}$, N. Oklay ${ }^{6}$, \\ M. Pajola ${ }^{28}$, L. Sabau ${ }^{31}$, C. Tubiana ${ }^{6}$, J.-B. Vincent ${ }^{6}$, and K.-P. Wenzel ${ }^{10}$
}

(Affiliations can be found after the references)

Received 23 February 2015 / Accepted 12 May 2015

\begin{abstract}
Context. Since August 2014, the OSIRIS Narrow Angle Camera (NAC) onboard the Rosetta spacecraft has acquired high spatial resolution images of the nucleus of comet 67P/Churyumov-Gerasimenko, down to the decimeter scale. This paper focuses on the Imhotep region, located on the largest lobe of the nucleus, near the equator.

Aims. We map, inventory, and describe the geomorphology of the Imhotep region. We propose and discuss some processes to explain the formation and ongoing evolution of this region.

Methods. We used OSIRIS NAC images, gravitational heights and slopes, and digital terrain models to map and measure the morphologies of Imhotep.

Results. The Imhotep region presents a wide variety of terrains and morphologies: smooth and rocky terrains, bright areas, linear features, roundish features, and boulders. Gravity processes such as mass wasting and collapse play a significant role in the geomorphological evolution of this region. Cometary processes initiate erosion and are responsible for the formation of degassing conduits that are revealed by elevated roundish features on the surface. We also propose a scenario for the formation and evolution of the Imhotep region; this implies the presence of large primordial voids inside the nucleus, resulting from its formation process.
\end{abstract}

Key words. comets: individual: 67P/Churyumov-Gerasimenko - methods: data analysis

\section{Introduction}

Cometary nuclei, formed in a cold environment far from the Sun, are excellent tracers of the formation and evolution processes of the solar system, making their study particularly relevant (e.g., Weidenschilling 2004). Their physical nature, composition, distribution, formation, and evolution are fundamental for understanding how planets formed and evolved, and ultimately how water was brought to Earth (e.g., Dones et al. 2004; Duncan et al. 2004; Hartogh et al. 2011). Since the formation of the solar system, comets have been affected by collisions, dynamical perturbations, irradiation processes, and thermal alteration, which have modified their physico-chemical properties and their orbits (e.g., Weissman et al. 2004; Bockelée-Morvan et al. 2004; Delbó et al. 2014). As a result of the sublimation of ices as the comet approaches the Sun, cometary activity is expected to be the main process altering the external layers of the nucleus. However, this process is still poorly understood, and we do not know to which extent it affects the surface and whether primordial unaltered regions still exist on the nucleus. The geomorphology of the nucleus provides key elements to address these questions.

Past studies on the geomorphology of cometary nuclei (1P/ Halley, 19P/Borrelly, 81P/Wild 2, 9P/Tempel 1, 103P/Hartley 2, and $67 \mathrm{P} /$ Churyumov-Gerasimenko) have revealed several types of terrains and geological features (Britt et al. 2004; Brownlee et al. 2004; Thomas et al. 2007, 2013a,b, 2015b; Basilevsky \& Keller 2007; Sierks et al. 2015). The variety of terrains includes smooth, mottled, pitted, and brittle terrains as well as terrains with exposed consolidated materials. Features include dark and bright spots, mesas, ridges, mounds, pits, and circular features, some of which are tentatively interpreted as impact craters. Layering has also been observed or at least suggested on 9P/Tempel 1 and 81P/Wild 2. Finally, flows have been proposed to explain the large smooth areas observed on Tempel 1 (Belton \& Melosh 2009). The origin and chronological evolution of most, if not all, of the terrains and geological features are still very uncertain, if not completely unknown.

The Rosetta mission from the European Space Agency is orbiting comet 67P/Churyumov-Gerasimenko (hereafter 67P) since August 2014. The Optical Spectroscopic and Infrared Remote Imaging System (OSIRIS; Keller et al. 2007), composed of two cameras, has acquired thousands of images of the nucleus surface with several filters in the visible, near-UV, and nearinfrared wavelength range $(250-990 \mathrm{~nm})$. This instrument offers a unique opportunity to study the geomorphology of a comet nucleus in detail and with unprecedented spatial resolution (up to the decimeter scale). 


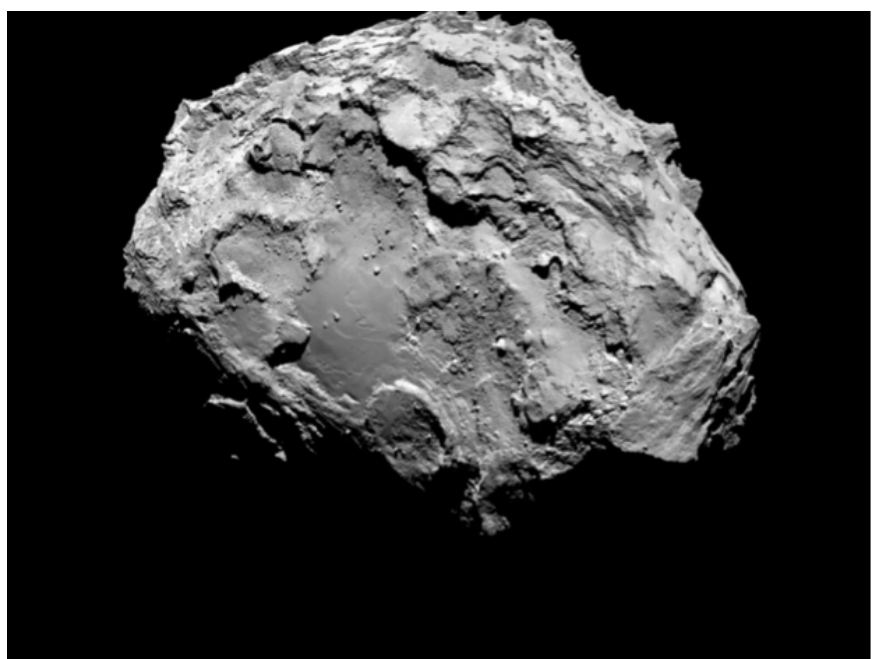

Fig. 1. Overview of the Imhotep region, located on the largest lobe of the nucleus, whose largest dimension is $4.1 \mathrm{~km}$. This image was acquired with the NAC camera on 3 Aug. 2014 from a distance of $282 \mathrm{~km}$. The spatial resolution is $5.3 \mathrm{~m} /$ pix. (NAC_2014-08-03T16.19.34.)

In this paper, we focus on the Imhotep region (Fig. 1), as defined in Thomas et al. (2015b) and El-Maarry et al. (2015), which is very interesting for various reasons. This region is located close to the equator and is relatively flat compared to the overall shape of the nucleus, so that it is illuminated daily from aphelion (5.7 AU) to perihelion (1.2 AU) and never enters polar night during the comet revolution around the Sun. It is a complex region with a broad diversity of geomorphological features, the most striking of which are a large smooth area of $0.8 \mathrm{~km}^{2}$ and several round features located in the gravitational lows. These particular illumination conditions and overall geomorphology render Imhotep a good candidate for being an active region when approaching perihelion (Keller et al. 2015). This point is further reinforced by the observations of Vincent et al. (2013), who suggested the equatorial region as the source of the strongest jet observed on 67P close to perihelion. Finally, Imhotep may be representative of the regions of the southern hemisphere that have not yet been illuminated and imaged, but will exit polar night in March 2015 and be fully illuminated at perihelion in August 2015.

The aim of this paper is to describe the geomorphology of the Imhotep region using the images of the OSIRIS cameras onboard Rosetta (Sects. 2 and 3 ) and to propose different scenarios and processes for the formation and evolution of the observed structures (Sects. 4 and 5).

\section{Data and tools}

\subsection{Images}

The OSIRIS instrument is composed of two cameras: a Narrow Angle Camera (hereafter NAC) and a Wide Angle Camera (Keller et al. 2007). This work is based on the analysis of the NAC images of the Imhotep region acquired from 3 August 2014 to 22 November 2014. For the regional mapping, we used images with a spatial resolution between $5.0 \mathrm{~m} /$ pix and $0.8 \mathrm{~m} /$ pix (Table 1), while for the local analysis we used images with a better spatial resolution, up to $34 \mathrm{~cm} /$ pix.

\subsection{Gravitational heights and slopes}

On Earth, the upslope and downslope directions are intuitive as gravity points towards the Earth's center to a very good approximation. On small bodies of a few kilometers such as 67P, the very irregular shape and the rotation strongly influence the shape of the equipotential lines, and the direction of slopes is no longer intuitive (Thomas 1993). On 67P, we calculated the gravity vector of a surface point by taking into account the gravity of the body itself, derived from its tridimensional shape (Gaskell et al. 2008; Jorda et al. 2014) assuming a homogeneous nucleus with a density of $470 \mathrm{~kg} / \mathrm{m}^{3}$ (Sierks et al. 2015), and the centrifugal force resulting from its rotation (Werner \& Scheeres 1996). To emphasize the role of the gravitational forces in the formation and evolution of the Imhotep morphologies, we used gravitational heights or "dynamic heights" as defined by Thomas (1993). The gravitational heights are calculated as follows (Vanicek \& Krakiwsky 1986; Thomas 1993):

$H=\frac{W_{1}-W_{0}}{g_{0}}$

where $W_{1}$ and $W_{0}$ are the gravitational potential energies per unit mass of the measured point $\left(W_{1}\right)$ and reference point $\left(W_{0}\right)$ and $g_{0}$ is the local gravity at the reference point.

\subsection{Tools}

The images and digital terrain models (DTM) were imported into the ArcGis 10.2 software. We used ArcGis for mapping and projections (e.g., Fig. 2), area and size measurements (e.g., Fig. 13) and downslope direction estimate (e.g., Fig. 14).

\section{Geomorphology of the Imhotep region}

The Imhotep region is located close to the equator and exhibits a wide variety of features that are different in texture, morphology, and photometric properties (Fig. 2). These variations hold clues to understanding the processes that shaped the surface and the underlying structure of the object. The first step to unravel these processes is to map the surface, grouping terrains with similar properties (landforms, texture, albedo) into morphological units that may have experienced a similar formation and evolution. The nature, location, and stratigraphic arrangement of the morphological units provide insights into their history and ultimately constrain the formation and evolution scenario of the comet.

We used six NAC images to map the Imhotep region. They were assembled to cover the entire region with a spatial resolution from $0.8 \mathrm{~m} /$ pix to $5.1 \mathrm{~m} /$ pix (Fig. $2 \mathrm{a}$ and Table 1). From this geomorphological mapping (Fig. 2b), we identified

- two types of terrains: smooth (Sect. 3.1) and rocky (Sect. 3.2),

- two specific areas: accumulation basins (Sect. 3.3) and bright spots (Sect. 3.4), and

- three remarkable morphological features: linear features (Sect. 3.5), roundish features (Sect. 3.6), and boulders (Sect. 3.7).

\subsection{Smooth terrains}

Smooth terrains are striking on Imhotep and are characterized by a material that is spatially unresolved in images with a pixel scale of $1 \mathrm{~m}$ (Fig. 2). However, images with a better spatial resolution of $34 \mathrm{~cm} /$ pix show a textured surface in some areas or directly allow detecting individual grains in other areas (Fig. 3). They show that the material in the smooth terrains consistes of relatively fine grains, with a size of up to a few tens of centimeters for the largest ones. There are spatial inhomogeneities in the size distribution of grains in the smooth areas. 
A.-T. Auger et al.: Geomorphology of the Imhotep region on comet 67P/Churyumov-Gerasimenko from OSIRIS observations

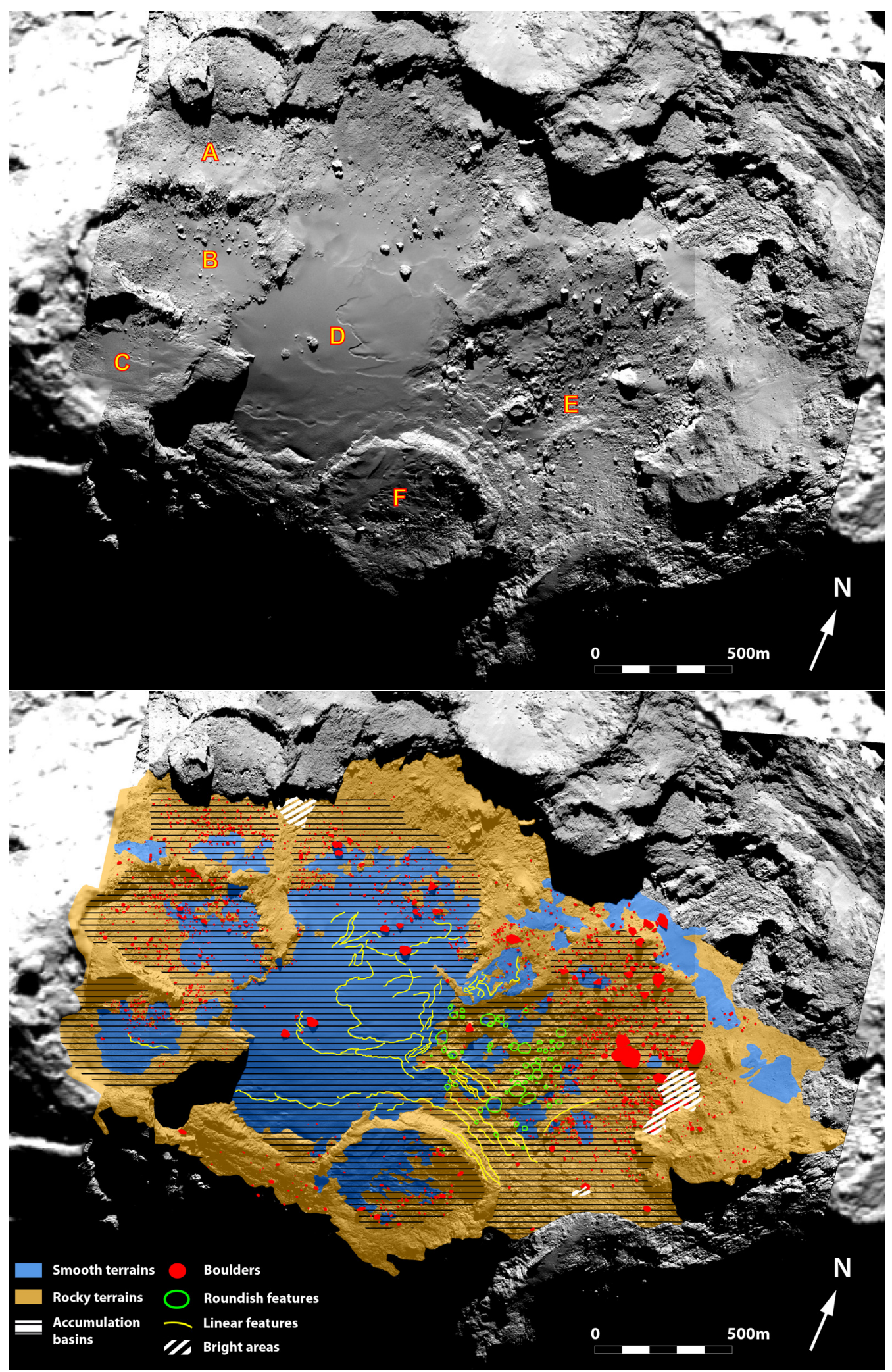

Fig. 2. Upper panel: mosaic of six NAC images covering the Imhotep region (see Table 1 for the list of images). Letters A to F are reference names for the accumulations basins (see text for details). Lower panel: geomorphological mapping of the Imhotep region.

Smooth terrains cover about one third of the Imhotep region. Most of the smooth terrains are located in the local gravitational lows (Fig. 4), and all of them are located in flat areas where the gravitational slope is lower than $15^{\circ}$ (Fig. 5). The largest smooth area, located at the center of the region, covers a surface of $0.8 \mathrm{~km}^{2}$. It is remarkably flat, with gravitational slopes lower than $5^{\circ}$ and very close to the gravitational lowest point of Imhotep (Fig. 4). Although less extended, some smooth terrains are also visible in the area of roundish features (Figs. 4 and 9) that are located in the gravitational low of the region. 
Table 1. NAC images used for the general mapping of the Imhotep region.

\begin{tabular}{lrcr}
\hline \hline Image & \multicolumn{1}{c}{ Date } & Distance to comet center $(\mathrm{km})$ & Pixel scale $(\mathrm{m})$ \\
\hline NAC_2014-08-03T17.19.34.549 & 3 Aug. 2014 & 273.3 & 5.1 \\
NAC_2014-08-25T23.12.54.550 & 25 Aug. 2014 & 52.1 & 1.0 \\
NAC_2014-09-05T06.31.16.575 & 5 Sept. 2014 & 43.2 & 0.8 \\
NAC_2014-09-05T06.35.55.557 & 5 Sept. 2014 & 43.2 & 0.8 \\
NAC_2014-09-05T06.40.55.560 & 5 Sept. 2014 & 43.1 & 0.8 \\
NAC_2014-09-05T06.45.55.557 & 5 Sept. 2014 & 43.1 & 0.8 \\
\hline
\end{tabular}

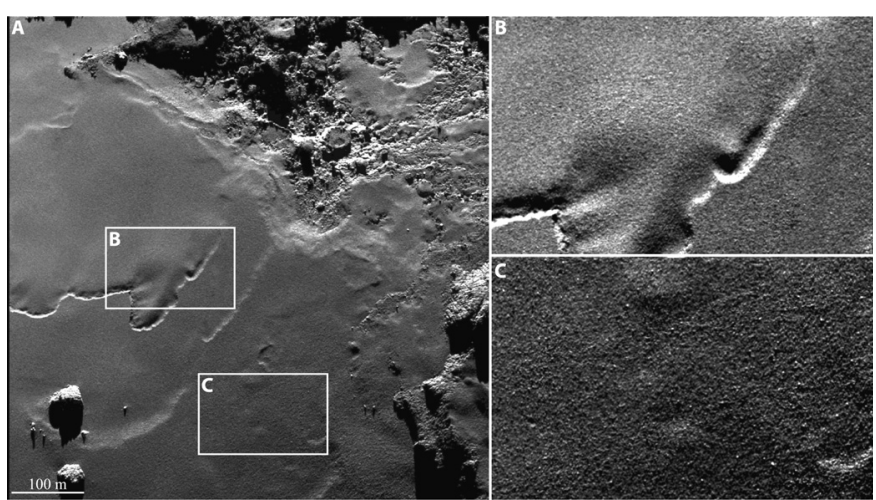

Fig. 3. Smooth terrains on Imhotep and zooms on the fine material for two different areas. This image was acquired with the NAC camera on 5 Oct. 2014 from a distance of $18 \mathrm{~km}$. The spatial resolution is $34 \mathrm{~cm} /$ pix. (NAC_2014-10-05T16.09.11.)

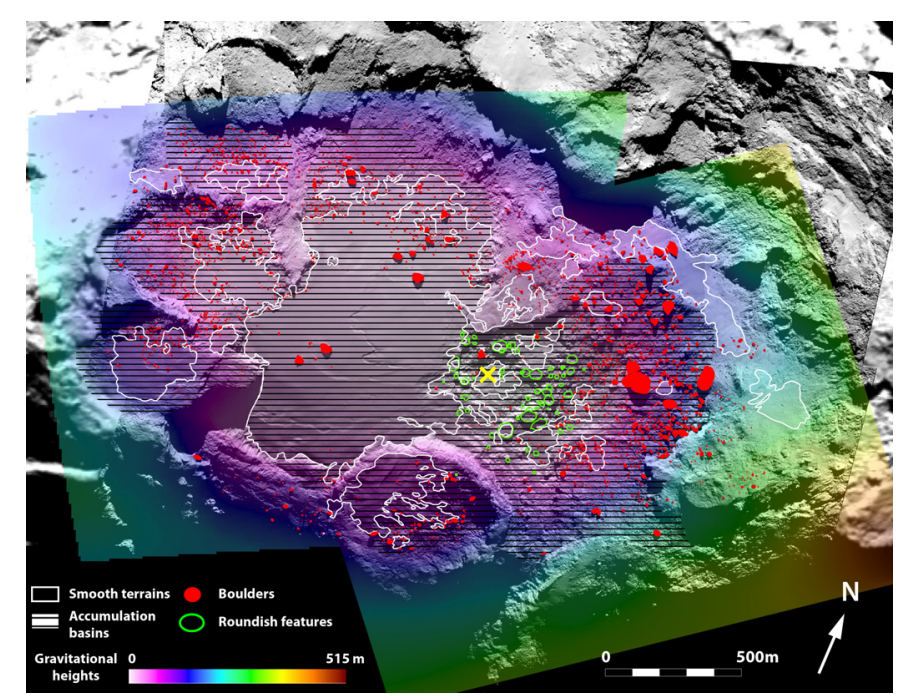

Fig. 4. Gravitational heights on Imhotep, shown in transparency above a mosaic image of the region. Accumulation basins, boulders, and smooth terrain boundaries are also represented. The yellow cross indicates the gravitationally lowest point of the region.

The thickness of smooth terrains seems to vary across the region, although we cannot quantify it. Upslope on the borders of the basins, the thickness of the fine material gradually decreases, revealing the underlying rocky terrains. Finally, smooth terrains are cut by terrace margins (Figs. 2 and 3) that can be gentle or very steep.

\subsection{Rocky terrains}

Rocky terrains are so called to distinguish them from smooth terrains. Rocky terrains consist of exposed, consolidated materials. As they are covered by a fine material in flat areas, most

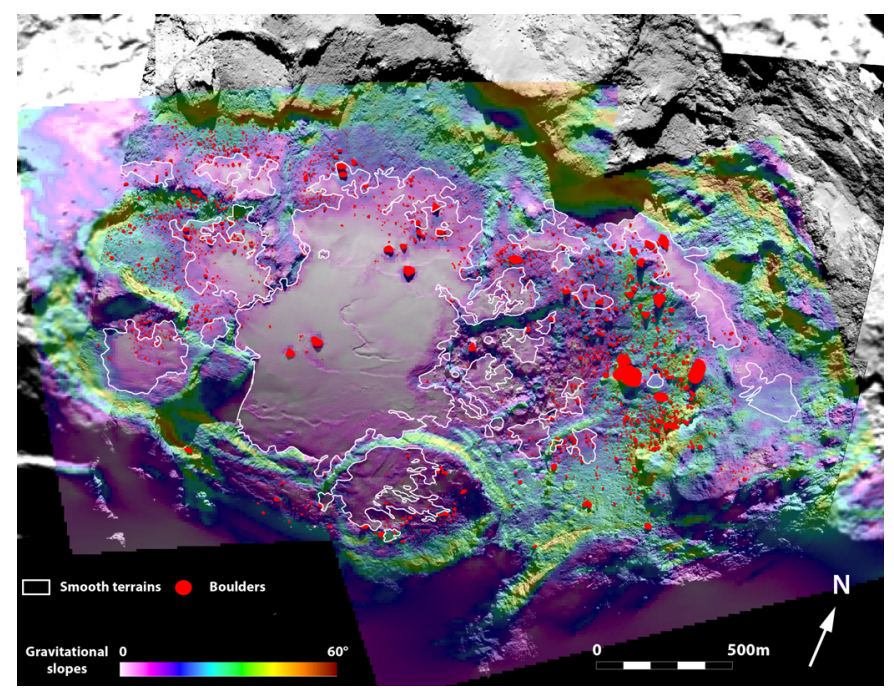

Fig. 5. Gravitational slopes on Imhotep, shown in transparency above a mosaic image of the region. Smooth terrain boundaries and boulders are also represented.

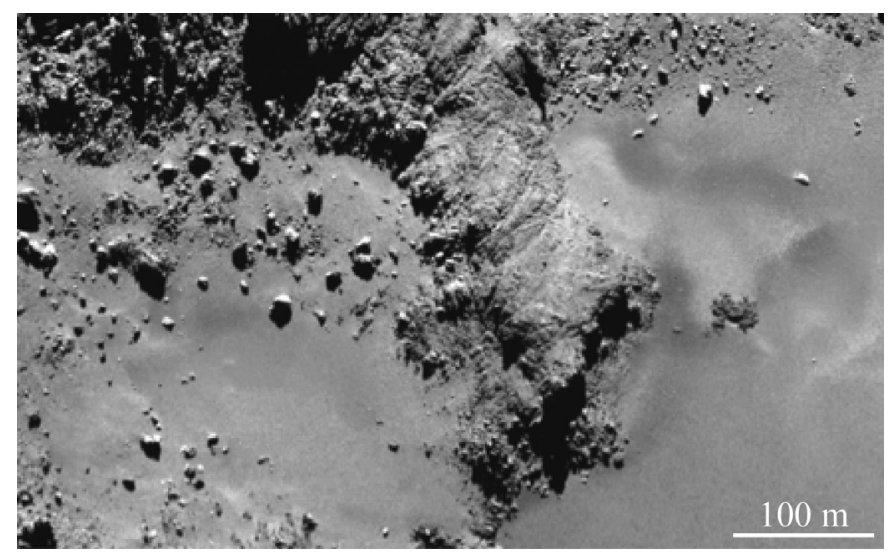

Fig. 6. Rocky terrains with fractures. This image was acquired with the NAC camera on 18 Sept. 2014 from a distance of $28 \mathrm{~km}$. The spatial resolution is $54 \mathrm{~cm} /$ pix. (NAC_2014-09-18T05.22.28.)

of them are only visible at the peripheries of the Imhotep region (Fig. 2), on high gravitational slopes, up to $60^{\circ}$ (Fig. 5). Rocky terrains on Imhotep are made of a highly fractured material. The fractures present different orientations (Fig. 6), which are sometimes locally associated with a feature (Fig. 18, Sect. 4.3). These fractures are quite common on the nucleus of $67 \mathrm{P}$ and have been observed in other regions (Thomas et al. 2015b).

The notable exception to these statements is the area of roundish features located in the gravitational low of the region (Fig. 4), where rocky terrains are still visible in a more chaotic arrangement and do not show fractures at the meter scale (Figs. 2 and 9). 

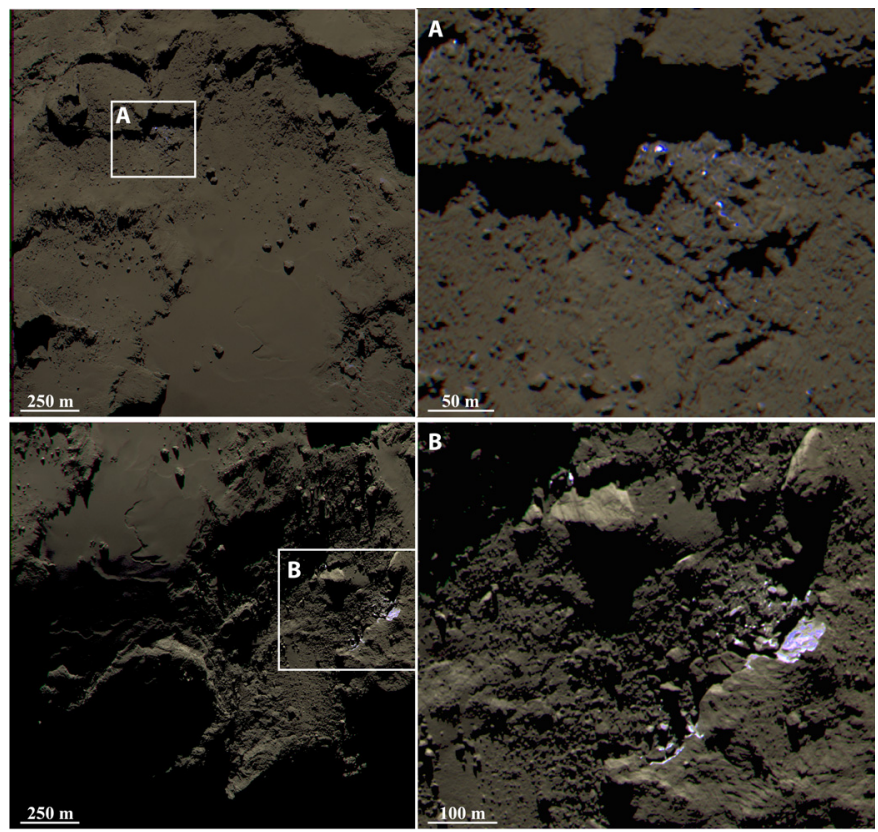

Fig. 7. Color-composite images with zoom on the bright, bluer, areas. We used three filters: blue $(480 \mathrm{~nm})$, green $(536 \mathrm{~nm})$, and orange $(649 \mathrm{~nm})$. The images were acquired with the NAC camera on 5 Sept. 2014 from a distance of $43 \mathrm{~km}$. The spatial resolution is $81 \mathrm{~cm} /$ pix. (NAC_2014-09-05T06.45 and NAC_2014-09-05T08.00.)

\subsection{Accumulation basins}

Morphologically, we define the accumulation basins as areas where a fine material and boulders seem to accumulate preferentially (Fig. 2). These basins correspond to the gravitational lows of the region (Fig. 4). They are all shaped like an alcove and have steep sides with gravitational slopes exceeding $40^{\circ}$. Except for basin $\mathrm{F}$ in the south, each basin opens toward a lower basin. We detected four minor basins of $0.1-0.2 \mathrm{~km}^{2}$ (basins A, B, C, and F) and two larger ones of $0.8 \mathrm{~km}^{2}$ each (basins D and E). Basin D corresponds to the largest smooth terrain and basin $\mathrm{E}$ to the area of roundish features. Accumulation basins cover twothirds of the Imhotep region and thus dominate the overall geomorphology of the region.

\subsection{Bright areas}

A few bright patches are visible in the images (Fig. 2). They are located at the basin edges. They are associated with boulders that have recently been illuminated (they were in the dark before due to large localized projected shadows from surrounding terrains), but also with a scarp east of the region that has been illuminated daily for several months (Fig. 7). These patches are bluer than the average color of the region, with a contrast of 7.5 at $550 \mathrm{~nm}$ ( $V$ band) between the dark and bright terrains (see Pommerol et al. 2015). This may be indicative of ice and water ice in particular (Sunshine et al. 2006; Pommerol et al. 2015). Water ice is also supported by the lack of changes of these bright areas over a period of several weeks, which excludes more volatile species such as $\mathrm{CO}_{2}$ ice (Pommerol et al. 2015). In the Imhotep region, bright areas represent less than $1 \%$ of the total surface area.

\subsection{Linear features}

The Imhotep region presents linear features as slope breaks (Fig. 2). These linear features are long compared to the size of the nucleus, typically hundreds of meters and up to $1 \mathrm{~km}$ for

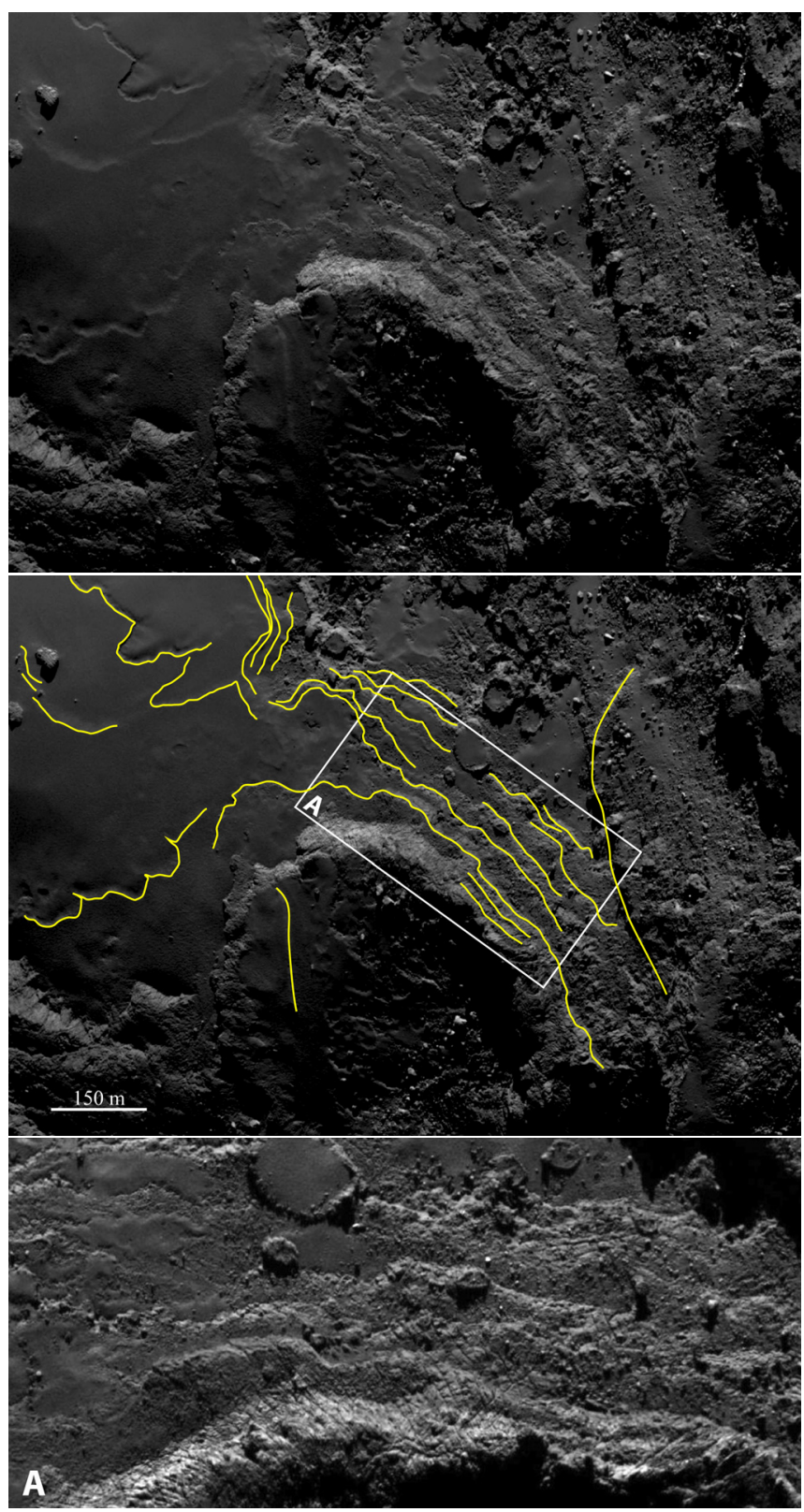

Fig. 8. Top and middle: linear features and terraces in the center of the Imhotep region. This image was acquired with the NAC camera on 5 Sept. 2014, from a distance of $43.5 \mathrm{~km}$. The spatial resolution is $80 \mathrm{~cm} /$ pix. (NAC_2014-09-05T05.21.16). Bottom: zoom on terraces cut by fractures. This image was acquired with the NAC camera on 16 Sept. 2014 from a distance of $28 \mathrm{~km}$. The spatial resolution is $50 \mathrm{~cm} /$ pix. (NAC_2014-09-16T01.17.56.)

the longest ones. All linear features are located near the gravitational low of the region, with the exception of one linear feature in basin C. Several linear features cross the interface of the rocky to the smooth terrain without discontinuities, suggesting a continuity of the rocky terrain topography below the smooth material (Figs. 2 and 8). In the vicinity of basin F, linear features correspond to roughly concentric scarps that can be described as terraces (Fig. 8). We identified about ten terraces, with a height of $5 \pm 2 \mathrm{~m}$ derived from the DTM (Jorda et al. 2014).

\subsection{Roundish features}

The roundish features identified in Fig. 2 (see also Fig. 9) are characterized by a circular, elliptical, or irregular roundish 


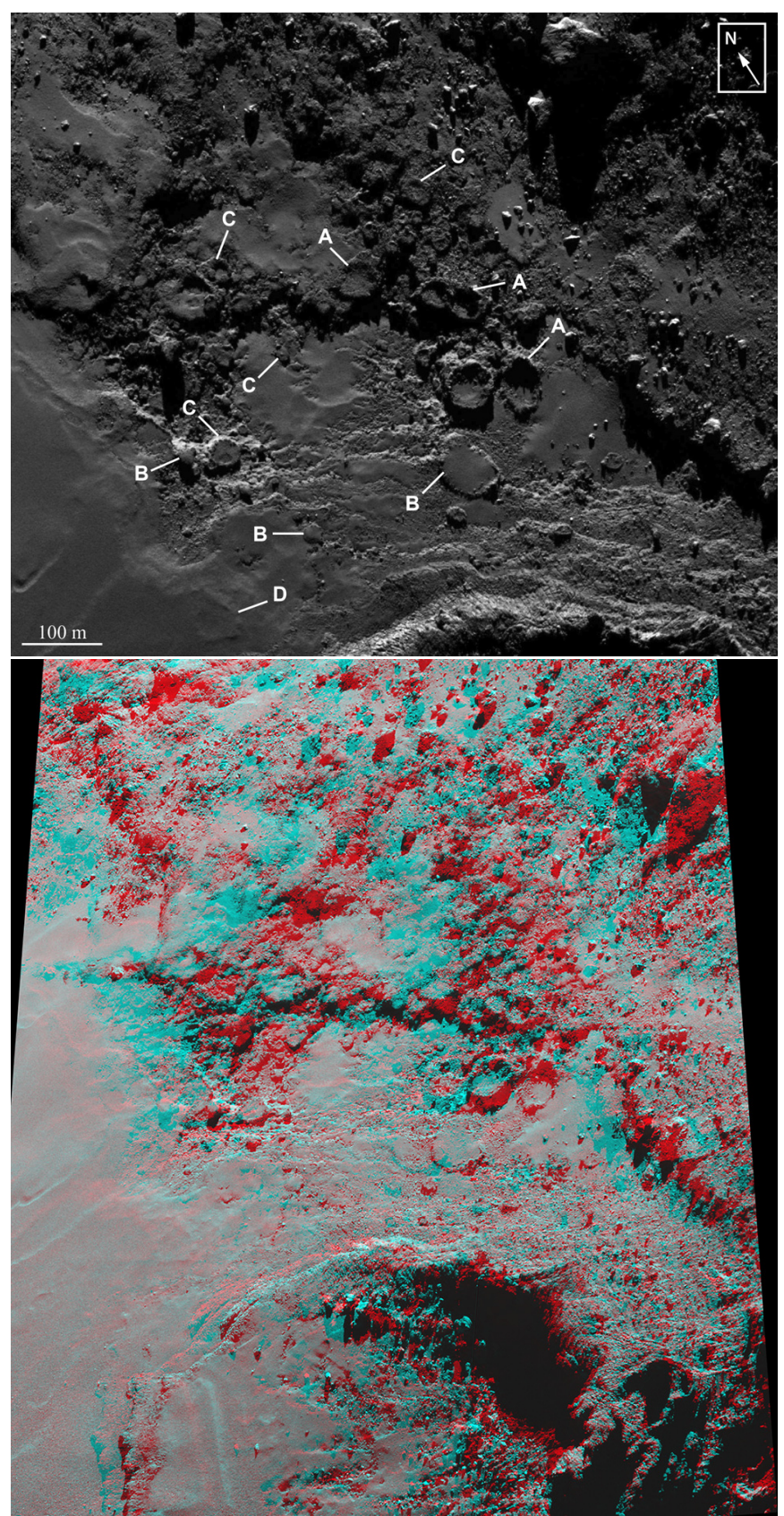

Fig. 9. Roundish features in the center of the Imhotep region. Upper panel: examples of roundish features: (A) with a rim and a depression on top, (B) with a rim and a fine-material mesa on top, sometimes bulging and (C) multiple roundish features that are nested in or are vertically stacked on top of each other, and (D) roundish feature covered by fine material. This image was acquired with the NAC camera on 16 Sept. 2014 from a distance of $28 \mathrm{~km}$. The spatial resolution is $50 \mathrm{~cm} /$ pix. (NAC_2014-09-16T01.17.56.) Lower panel: anaglyph of the roundish feature area from two NAC images acquired on 22 Nov. 2014 from a distance of $31 \mathrm{~km}$. The spatial resolution is $56 \mathrm{~cm} / \mathrm{pix}$. (NAC_2014-11-22T06.52.)

shape, elevated relative to the surroundings. They are located in the gravitationally lowest area of the Imhotep region and have not yet been observed in any other region of 67P (Thomas et al. $2015 b)$. They present a rim and at their top either a depression (Fig. 9, type A) or a mesa of a fine material that sometimes forms a bulge (Fig. 9, type B). Many of these roundish features appear in groups that are nested in or are vertically stacked on top of each other (Fig. 9, type C). A few roundish features appear to

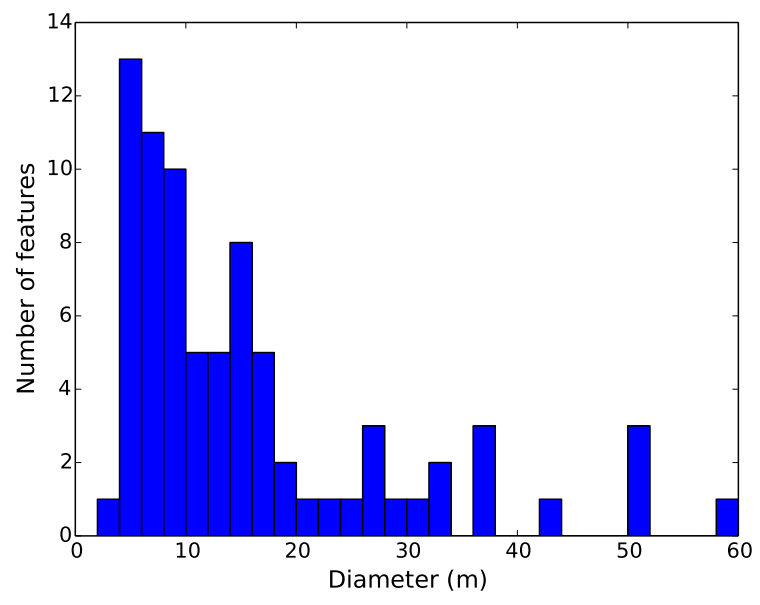

Fig. 10. Size distribution of roundish features. We counted more than 70 , with sizes between $2 \mathrm{~m}$ (lower limit with our images) and $59 \mathrm{~m}$.

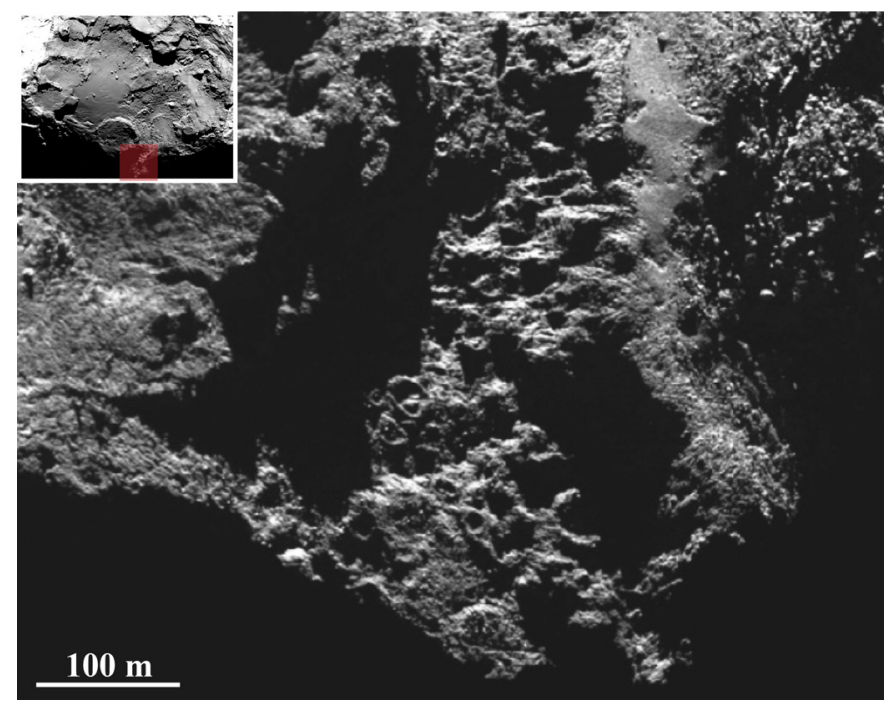

Fig. 11. Regions in the south of Imhotep that begin to be illuminated, revealing additional roundish features. This image was acquired with the NAC camera on 31 Oct. 2014 from a distance of $33 \mathrm{~km}$. The spatial resolution is $63 \mathrm{~cm} /$ pix. (NAC_2014-10-31T14.19.35.)

be covered by a fine material (Fig. 9, type D). Some roundish features are also located on the terraces and more particularly on the margins of the terraces close to basin F. From south to north, roundish features seem to be more degraded and less filled with a fine material.

With a spatial resolution of $50 \mathrm{~cm} /$ pix (Fig. 9), we identified more than 70 roundish features, with sizes (diameter) between $2 \mathrm{~m}$ (lower limit with our images) and $59 \mathrm{~m}$ (Fig. 10). Their size distribution is neither flat nor Gaussian or logarithmic, so that there does not seem to be a characteristic size for these features. The general trend is a decrease of their number for larger sizes, but this is subject to caution because of the low statistic.

Finally, as 67P approaches perihelion, the southern part of Imhotep starts to be illuminated, and additional roundish features become visible (Fig. 11).

\subsection{Boulders}

Similar to many other regions on 67P (El-Maarry et al. 2015; Pajola et al. 2015), Imhotep shows many boulders, with sizes 
A.-T. Auger et al.: Geomorphology of the Imhotep region on comet 67P/Churyumov-Gerasimenko from OSIRIS observations

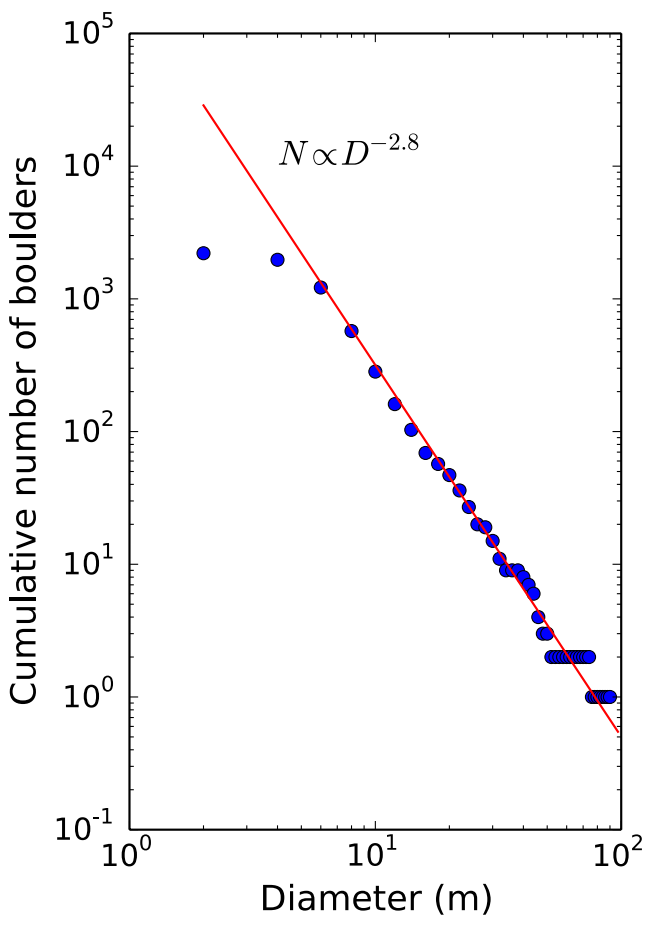

Fig. 12. Cumulative size distribution of 2207 boulders, which follows a power law with an exponent of $2.8 \pm 0.1$.

(diameter) from $2 \mathrm{~m}$ (lower limit with our images) to $90 \mathrm{~m}$. We counted 2207 boulders in Fig. 2. Their cumulative size distribution is shown in Fig. 12 and follows a power law with an exponent of $-2.8 \pm 0.1$. It is complete down to $6 \mathrm{~m}$ in size.

Boulders are mainly located on the slopes surrounding the regional gravitational low, and more particularly on southward slopes in the western half of the region (Figs. 4 and 5). Their slope distribution peaks around $10^{\circ}$, and most boulders are located on intermediate slopes between $5^{\circ}$ and $25^{\circ}$. There are no boulders on slopes steeper than $50^{\circ}$, and $98 \%$ of them are located on gravitational slopes lower than $35^{\circ}$ (Fig. 13, upper panel). There are very few boulders on smooth and flat terrains (slope $<3^{\circ}$ ). Finally, there is no obvious correlation between the size of the boulders and the gravitational slopes of the terrain on which they stand (Fig. 13, lower panel).

The boulders differ not only in size, but also in texture. They could either be conglomerate or highly fractured (Fig. 15). If they are fractured, fractures cross each other and do not have a specific and unique orientation.

\section{Discussion: geomorphology and processes}

\subsection{Smooth terrains}

Smooth terrains can be considered as relatively undisturbed areas that evolve slowly and where material has time to settle and accumulate. No fracture cuts through the smooth terrains as it does in rocky terrains, which suggests that the smooth terrains are more recent than the fractures or that they consist of loose material that is unable to retain fractures.

The erosion of rocky terrains and boulders may form the fine material constituting the smooth terrains. This eroded material may originate from different regions on the nucleus: a) it can be formed in situ, at the current location of the smooth terrain; b) it can be transported by gravity from the borders of the Imhotep basins; c) or it can be air-fall deposits from the coma following
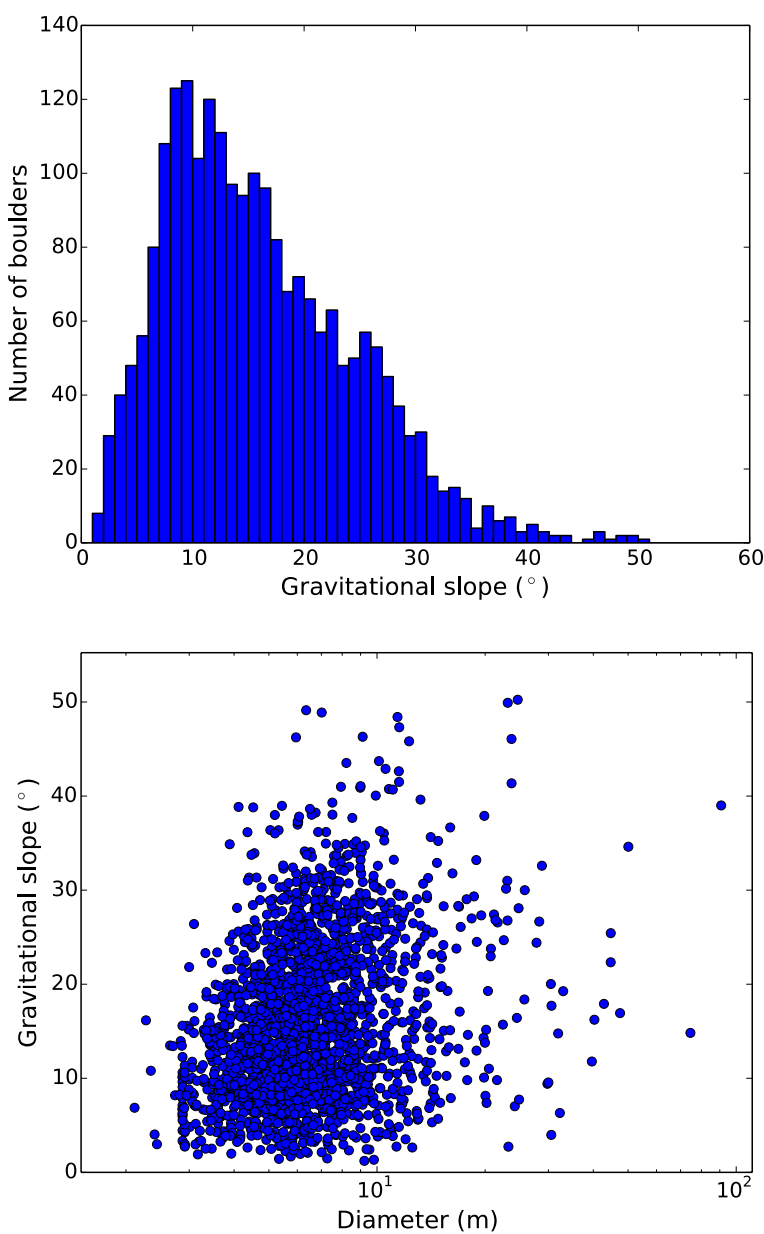

Fig. 13. Upper panel: histogram of the number of boulders as a function of the gravitational slope of the terrain on which they stand. Lower panel: gravitational slope of the terrain on which boulders stand as a function of their size.

an ejection processes in any other part of the nucleus. We explore here several processes related to these different origins.

Thermal fatigue - smooth terrain on small bodies is not uncommon and has already been subject to several interpretations. An explanation for the ponds on Eros is the in situ erosion of boulders by thermal fatigue (Dombard et al. 2010). The ponds and boulders in question are located at or near the equator of Eros, similar to Imhotep on 67P, where the diurnal thermal cycles are the strongest. However, smooth terrains around boulders on Eros do not extend very far, typically a few boulder radii. It therefore seems unlikely that the large amount of fine material on Imhotep is entirely due to thermal fatigue.

Dust levitation - Colwell et al. (2005) adopted the idea of dust levitation reported by Lee (1996) to provide another explanation for the formation of dust ponds on Eros. Dust is transported in photoelectron layers and redeposited in shadow areas or trapped in gravitational lows. If applicable to $67 \mathrm{P}$, this process only applies to particles smaller than $\sim 1 \mu \mathrm{m}$ and thus only concerns a small fraction (in mass) of the observed smooth terrain, which includes larger particles up to the decimeter scale.

Air-fall deposits - on 67P, the sublimation process leads to the ejection of particles that fall back onto the surface if they do not reach the escape velocity. Their fall is guided by the gravitational attraction and is then oriented toward the nearest gravitational low. However, this transport mechanism is limited to boulders smaller than the meter scale because larger boulders are 


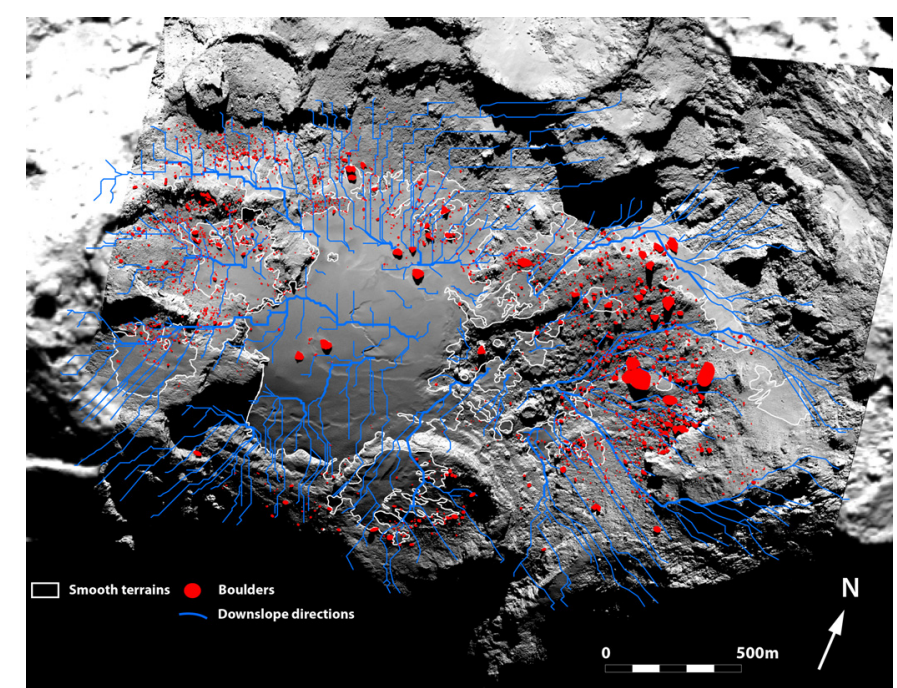

Fig. 14. Mosaic of the Imhotep region with smooth terrains, boulders, and downslope directions drawn from the gravitational heights with the ArcGis software.

too big to be lifted (Groussin \& Lamy 2003; Kelley et al. 2013; Pajola et al. 2015; Thomas et al. 2015a). Since many boulders larger than one meter are visible on Imhotep, including some on smooth terrains, air-fall deposits cannot account for all of them. Nevertheless, transport across the comet nucleus remains a likely process for small boulders $(<1 \mathrm{~m})$, pebbles, and finer material. With a dust and gas production rate of $255 \mathrm{~kg} / \mathrm{s}$ at perihelion and assuming that $50 \%$ of the dragged material falls back on the surface, a dust layer of $5 \mathrm{~cm}$ will cover the entire surface of the nucleus in two months at perihelion. This deposit is most likely distributed inhomogeneously on the surface, but it is certainly not negligible.

Seismic shaking - in Itokawa and Eros, seismic shaking remains the main mechanism to explain the presence of smooth terrains in gravitational lows (Richardson et al. 2004, 2005; Miyamoto et al. 2007). Seismic shaking tends to fluidize the material and to sort grains by size, with the finest grains migrating, by gravity, toward gravitational lows. However, considering the very low density of $470 \mathrm{~kg} / \mathrm{m}^{3}$ of $67 \mathrm{P}$ and its high porosity of 60-70\% (Sierks et al. 2015), the seismic energy is substantially attenuated compared to Itokawa and Eros (Love et al. 1993), whose densities are estimated at $1950 \mathrm{~kg} / \mathrm{m}^{3}$ (Abe et al. 2006) and $2640 \mathrm{~kg} / \mathrm{m}^{3}$, respectively (Thomas et al. 2002). Moreover, seismic shaking is usually triggered by a violent energetic event such as an impact, but no large impact crater has been unambiguously identified on the surface of 67P so far (Thomas et al. 2015b). This makes this process unlikely.

Fluidization of material - for comet 9P/Tempel 1, Belton \& Melosh (2009) invoked a fluidization process to explain the observed smooth terrains. The source of the flow would be a depression formed after the collapse of a cavity filled with gas. The escape of the gas causes the cometary material to fluidize; it is then transported by gravity to the gravitational lows. There are interesting similarities between the two comets: just like 9P/Tempel 1, the smooth terrains in Imhotep are restricted to the gravitational low. They are also adjacent to depressions (basins), and terraces and linear features may be interpreted as successive flows. Figure 14 shows the downslope directions on Imhotep calculated from the gravitational heights. It is clear that any material transported by gravity will end in smooth terrains. However, unlike smooth terrains on $9 \mathrm{P} /$ Tempel 1 , there is no evidence of flow features in this area. This is a strong limitation that makes the fluidization of material and flows not fully satisfying for Imhotep.

Transport by gravity - The only hint of a displacement of the fine material is located on the northern border of smooth terrains, at the interface between the smooth and rocky terrains, where the boulders and the fine material seem to interact (Fig. 15). The general direction appears to be downslope, as expected for a gravity process. Segregation in size seems visible, the finest material being located downhill. Following these observations, dust originates in the north and moves downslope toward the south until it is stopped by boulders and accumulates.

Based on this discussion, we propose the following scenario for the formation and evolution of smooth terrains on Imhotep: the fine material comes from the cliffs on the border of the basins where mass wasting occurs. It is then transported by gravity downslope to a flat surface where it stays. The wideness of the smooth area can be explained by the progressive retreat of the cliffs over a long time, probably some tens to hundreds of perihelion passages. The more distant the fine material from the cliff, the older the deposit is. We suggest that the linear features visible in smooth terrains reveal the topography of the rocky terrain underneath. Some might also be scars of the previous location of the cliffs. This scenario is more suitable with an erosion of cliff runs by events limited in time, such as the passage at perihelion that strongly increases the activity of the comet. Air-fall deposits are also not excluded, but probably only account for a small fraction of the smooth terrains.

\subsection{Rocky terrains}

While smooth terrains can be considered as the erosion product of rocky terrains, the latter are then the exposed part of the nucleus bedrock. Currently, we know little about this material. The bulk density of 67P's nucleus is very low and has been evaluated to be $470 \pm 45 \mathrm{~kg} / \mathrm{m}^{3}$, which can be explained by a high porosity of $60-70 \%$ (Sierks et al. 2015). The upper layer of rocky terrains is highly fractured and almost entirely depleted in volatiles, as suggested by the VIRTIS infrared spectroscopic observations that did not reveal water ice on the surface (Capaccioni et al. 2015). This is also confirmed by OSIRIS color images that showed a spectrally red and very homogeneous terrain across the Imhotep region (Fornasier et al. 2015).

Rocky terrains experience erosion, as emphasized by mass wasting that consists of boulders and finer material. Different processes can drive this erosion: a) fracturing; b) sublimation; c) outburst; and d) gravity processes.

Fracturing - as illustrated in Fig. 6, fractures on exposed rocks have different orientations that cannot be related to clearly identified structures. Moreover, since fractures are visible everywhere on the nucleus, from the hundred meter scale to the decimeter scale (Thomas et al. 2015b), it seems unlikely that they have been created by a single, catastrophic event such as an impact. The fractures on Imhotep at the meter scale might result from thermal fatigue and thermal shock, which are well known to affect rocks on airless bodies (Tauber \& Kuhrt 1987; Dombard \& Freed 2002; Dombard et al. 2010; Molaro \& Byrne 2012). As Pochat et al. (2009) phrased it, "this near-surface process may help to speed up other processes like mass wasting and sublimation degradation".

Sublimation - the second possible erosion process is the sublimation of ices, known to be the main driver of cometary activity and responsible for the nucleus erosion. Imhotep, located close to the equator, is illuminated every day over the entire comet revolution around the Sun. However, sublimation 

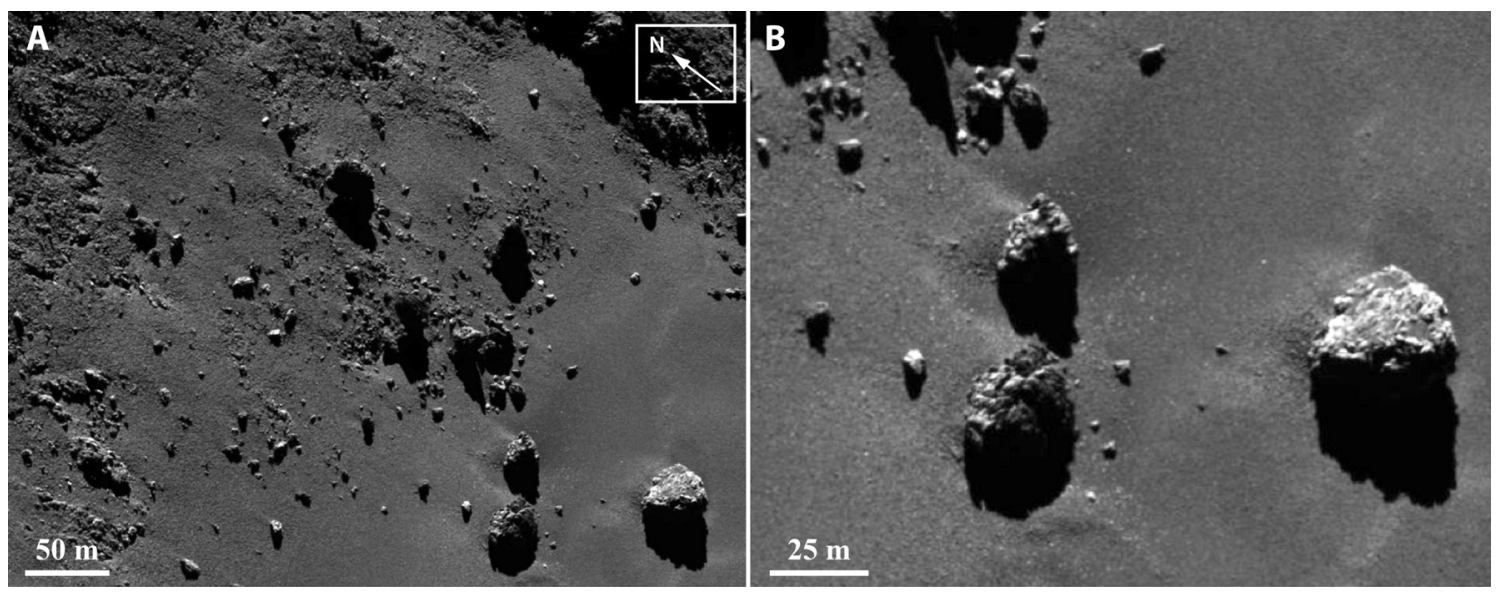

Fig. 15. A) Mass wasting on the northern border of Imhotep showing evidence of transport of the fine material. B) Zoom that shows textured boulders of tens of meters that stop the fine material from moving downslope. This image was acquired with the NAC camera on 5 Oct. 2014 from a distance of $18 \mathrm{~km}$. The spatial resolution is $34 \mathrm{~cm} /$ pix (NAC_2014-10-05T04.09.24.)

is stronger at perihelion when insulation, and therefore erosion, is highest. It is therefore likely that the mass wasting observed at 3 AU on Imhotep is just the beginning of an erosion phenomenon that will increase up to perihelion at $1.2 \mathrm{AU}$. Quantitatively, we expect surface erosion to be ten times stronger at $1.2 \mathrm{AU}$ than at $3 \mathrm{AU}$, if it is driven by water ice sublimation.

Outbursts - sporadic events such as outbursts can also erode the nucleus and even break it in the most catastrophic scenario (Boehnhardt 2004). Several small and short (a few hours) outbursts were observed by the Deep Impact spacecraft on comet 9P/Tempel 1 during the weeks preceding the flyby (A'Hearn et al. 2005). On comet 67P, an outburst was observed by OSIRIS on 30 April 2014, at 4.1 AU from the Sun, which lasted ten days (Tubiana et al. 2015). This outburst released $10^{3} \mathrm{~kg}$ to $10^{5} \mathrm{~kg}$ of material, which corresponds to an excavated volume equivalent to a sphere smaller than $15 \mathrm{~m}$, assuming a density of $470 \mathrm{~kg} / \mathrm{m}^{3}$ (Sierks et al. 2015). Such events, if they become more frequent as 67P approaches the Sun, could thus play an important role in eroding the nucleus surface and Imhotep in particular.

Gravity processes - when a rocky terrain has been eroded by sublimation and/or fragmented by fractures, gravity can modify it by mass wasting or collapse that is due to subsurface internal voids. The creation of overhangs by sublimation of a lower layer is a simple mechanism for mass wasting, and these overhangs have been observed in several places on the nucleus (Thomas et al. 2015b; Pommerol et al. 2015). Mass wasting on the northwestern part of Imhotep, clearly associated with the surrounding scarps, is a good candidate for an overhang (Fig. 16).

To summarize, the most likely scenario for the erosion of rocky terrains is that it is triggered by the sublimation of ices, controlled by gravity, and probably exacerbated by fractures.

An interesting question is whether rocky terrains, which are eroding, are also forming? Does an Earth-like rock cycle exist? We already know that if there is indeed a cycle, it can only be an open cycle since 67P loses some rocky material (i.e., dust) at each perihelion passage. Dust falling back onto the nucleus surface may re-form a rock, as it is the case for sediments on Earth. This requires a compaction mechanism or a cement/matrix between the grains to obtain a coherent material. The accumulation of material in a basin may lead to the compaction of the deepest layers. In this case, the new rocky terrain would no longer be made of primordial material, but of remobilized material from an older eroded rocky terrain. Such a rocky cycle is currently purely speculative.

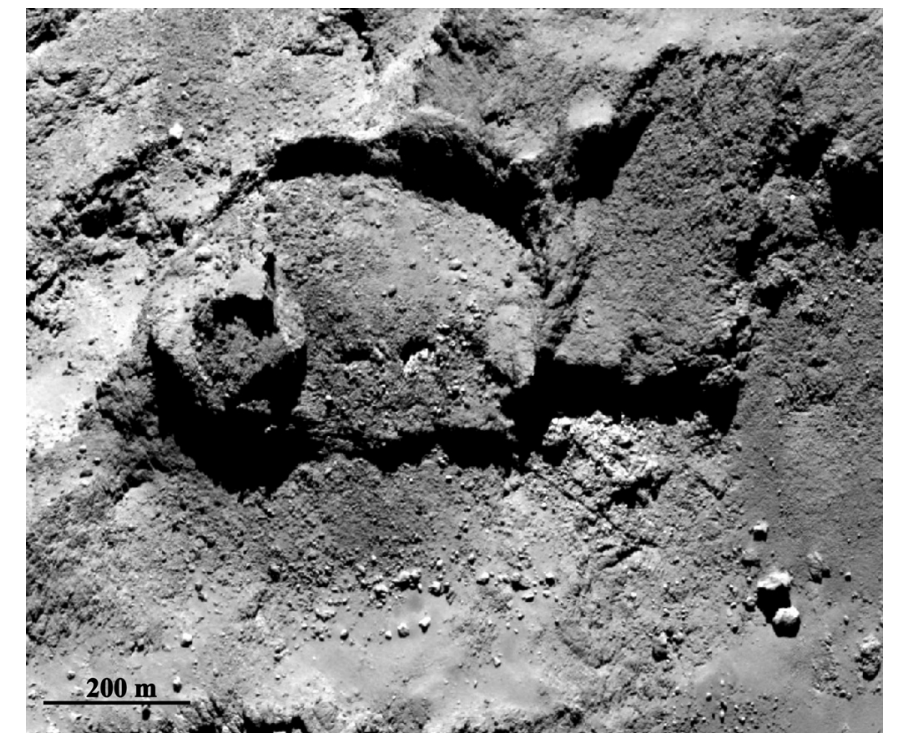

Fig. 16. Mass wasting and overhangs in the northwest of Imhotep. (NAC_2014-09-05T06.45.55.)

\subsection{Accumulation basins}

Accumulation basins are areas where the products of the erosion of rocky terrains will accumulate if they have not escaped the comet gravitational attraction for the finest grains. The boulders at the feet of the cliffs, on the borders of the basins, show that cliffs indeed erode and then retreat, as proposed before. The alcove shape of basins could result from their initial circular shape and/or from an erosion process. There have been several propositions to explain circular features, including pits, on other comets: a) impact craters; b) locally enhanced cometary activity; c) or collapse of a subsurface cavity.

Brownlee et al. (2004) and Vincent et al. (2014) were able to retrieve the shape of flat-floor pits and pit-halo features on 81P/Wild 2 from experimental and numerical simulations of impact craters. Thomas et al. (2007) considered that circular rim remnants and isolated rimless depressions on 9P/Tempel 1 are consistent with impact craters, although they were unable to prove their origin. An impact origin for the formation of some basins cannot be completely ruled out.

On the other hand, Belton et al. (2013) and Thomas et al. (2013a) ascribed the majority of pits and depressions observed 


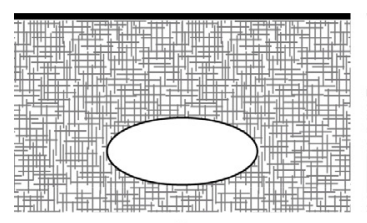

A

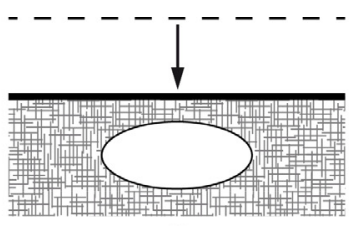

B

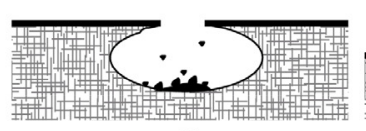

C

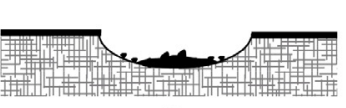

D

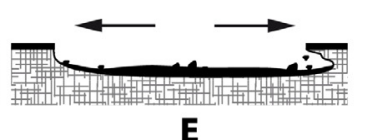

$\mathbf{E}$

Fig. 17. Formation and evolution of the accumulation basins on Imhotep. A) Primordial void into the nucleus, resulting from its formation process. B) Progressive erosion of the surface and thinning down of the layer of material above the void. C) and D) collapse of the thin upper layer. E) Enlargement of the basin and infill with eroded material of the edges. Possible formation of overhangs.
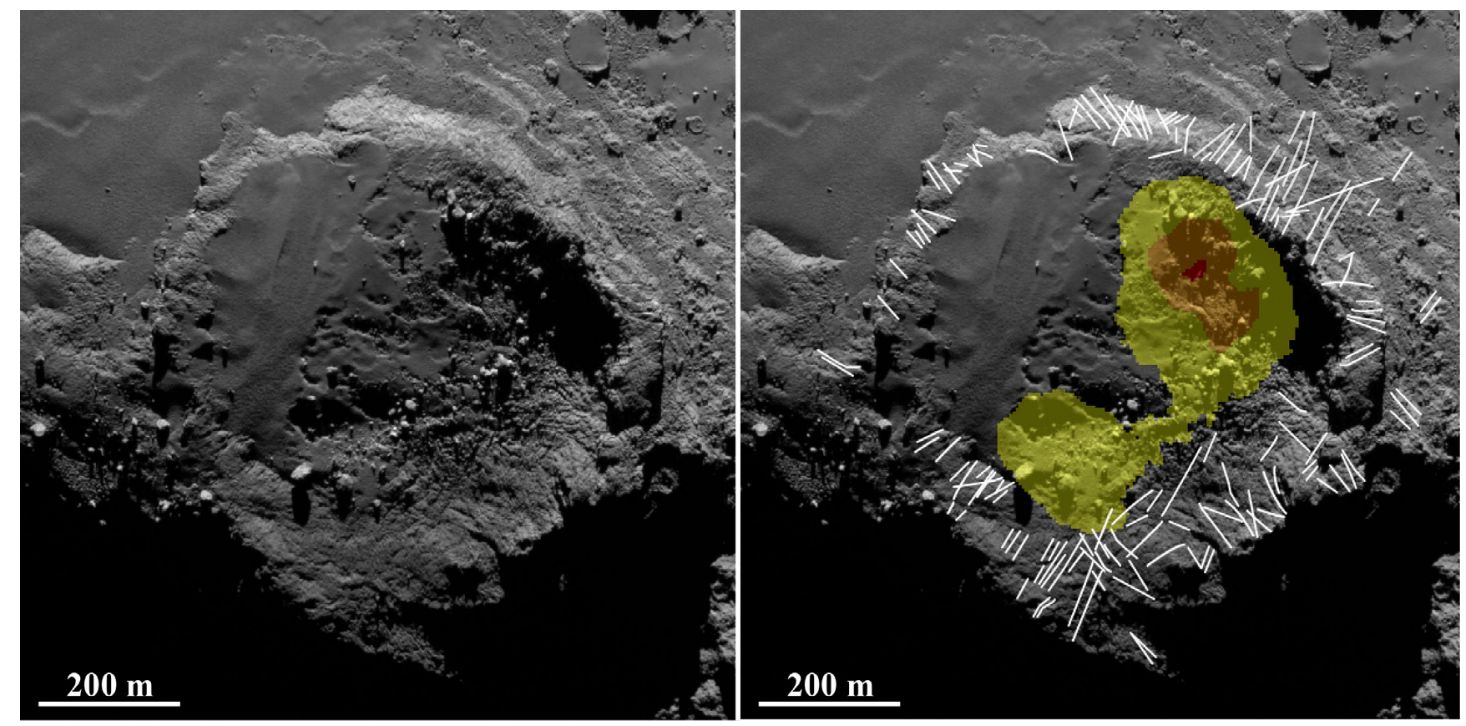

Fig. 18. Basin $\mathrm{F}$ and its fractures (white lines). The colored area represents the density of intersection points between prolongated fractures: the density increases from yellow to red. (NAC_2014-09-05T06.31.16.)

on 9P/Tempel 1 to endogenic processes such as outbursts. Belton \& Melosh (2009) also proposed that the origin of some large round features might be the collapse of a subsurface cavity, after the voiding of a gaseous material. However, this hypothesis relies on the transition from amorphous to crystalline water ice, which is exothermic and leads to the sublimation of the surrounding $\mathrm{CO}$ and $\mathrm{CO}_{2}$ ices. This process remains speculative because transferring solar energy from the surface to tens of meters into the nucleus is difficult to reconcile with the low thermal inertia of the nucleus of $10-50 \mathrm{~J} / \mathrm{m}^{2} / \mathrm{K} / \mathrm{s}^{1 / 2}$ (Gulkis et al. 2015). Indeed, the seasonal heat wave penetrates less than $1 \mathrm{~m}$ per revolution, and at $10 \mathrm{~m}$ depth the material is thermally isolated from the surface.

Our best interpretation for the formation of basins A to $\mathrm{E}$ remains the presence of large primordial voids into the nucleus, resulting from its formation process. After the surface above the voids is made fragile by erosion and fracturing, it can eventually collapse (Fig. 17). Primordial internal voids must have a volume equivalent to the formed basin. The resulting basin can then extend radially, with erosion. This scenario implies that the size of the current basins may be significantly larger than that of the initial basins formed just after collapse.

Basin $\mathrm{F}$ appears to be different from the other basins since it shows many fractures that point radially toward its interior (Fig. 18). Radial fracturing results from mechanical stress that cannot be triggered by a gravitational process such as a collapse, since it will only affect the collapsed material and not its surroundings. Two processes previously mentioned may thus be at the origin of these fractures and of basin F: 1) impact cratering or 2) elevation by the rising up of a gas bubble from the interior of the nucleus triggererd by cometary activity. It is currently not possible to favor one scenario over the other.

\subsection{Terraces}

The terraces on Imhotep and on the nucleus in general (Thomas et al. 2015b) strongly suggest layering (Massironi et al. 2015). Layers can be material of different compositions or compaction and/or successive deposits. There is no variation in color or albedo between the layers, but an important observation is that they have a relatively constant thickness of a few meters, which implies a repetitive process. Layers may be primordial, resulting from the formation process of the nucleus (Belton et al. 2007), or formed later by evolutionary processes (Belton \& Melosh 2009).

The fractures of basin F cross several terraces around it (Figs. 8 and 18), indicating that fractures are posterior to the formation of the layers. Chronologically, layers were formed before basin F. However, we cannot prove whether or not they are primordial.

\subsection{Roundish features}

In the center of Imhotep, the region of roundish features presents clear characteristics. In addition to the fact that it is the only place so far on the nucleus where this type of features has been detected, this region is

- located in the lowest part of the Imhotep region in terms of gravitational heights (basin E),

- depleted in boulders, 
- depleted in fine-material deposits in contrast to all other gravitational lows on Imhotep and on the nucleus, and it is

- located close to terraces. Processes that can form round features on the surface are a) impact cratering; b) collapsing; or c) cometary activity.

Impact cratering can explain the rim observed for the roundish features, with the compression induced by this process, but it cannot explain the cylindrical shape or the bulging top of some roundish features. In a similar way, the collapse of a primordial cavity cannot explain the cylindrical rimmed shape. These two processes, impact cratering and collapsing, seem excluded. We remain with cometary activity as the most plausible process that formed the roundish features.

Brownlee et al. (2004) proposed that the cylindrical shape of the roundish features might be an ancient gas conduit consolidated by the deposit of harder particles like water ice on its walls. Walls have been exposed to the surface following the erosion of the surrounding terrains. Belton \& Melosh (2009) developed this idea of gaseous conduit with their concept of a "spouting flow" of $\mathrm{CO}$ and $\mathrm{CO}_{2}$ gas rising up from the comet interior to the surface and leading to outbursts.

Roundish features are located close to terraces, suggesting that they are closely linked. Following the idea of Brownlee et al. (2004), we propose that roundish features are indeed ancient degassing conduits that have been revealed by the differential erosion of pre-existing layers (Fig. 19). Their consolidated walls allow their shape to be preserved and also prevent the retreat of the layers, which explains why some roundish features are located at the margins of terraces and why some are only barely visible (type D in Fig. 9). The erosion explains the depletion in fine material and boulders in this region. Northern roundish features are more degraded, which indicates that they may have been exposed for a longer time to solar illumination. Moreover, terraces are orientated toward the north. These two observations suggest that the erosion front proceeds from north to south. The source of gas at the origin of the conduit formation and the reason why the erosion is - or was - more efficient in basin E are still unclear, but the passage of the comet at perihelion may bring us answers.

Finally, we also mention the strong similarity of the roundish features on Imhotep with those observed on the nucleus of comet 9P/Tempel 1 (Fig. 20). They share similar morphological properties, are all located in a gravitational low and have similar sizes, from some tens of meters to $350 \mathrm{~m}$ for the largest ones (Thomas et al. 2007).

\subsection{Bright areas}

If bright patches, which are bluer than their surroundings, do indeed reveal the presence of water ice (Pommerol et al. 2015; Capaccioni et al. 2014), bright areas should be the youngest part of the region, as they are the most unstable. The presence of bright patches further reinforces the idea that the basins have been enlarged by the progressive retreat of their borders; this retreat is more efficient at the perihelion passage since it is driven by sublimation. Figure 7 shows that the bright areas are indeed associated with mass wasting, but also with collapsing pieces of the surrounding rocky terrain. From these observations, and in particular because of the icy scarp visible in the east-southeast of the region (Fig. 7, bottom), it seems likely that most if not all rocky terrains do contain a large fraction of water ice, hidden under a layer depleted in volatiles. It is not possible, however, to estimate the amount of water ice and the thickness of the depleted layer from our observations.

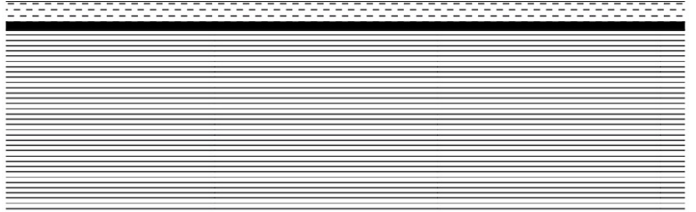

A

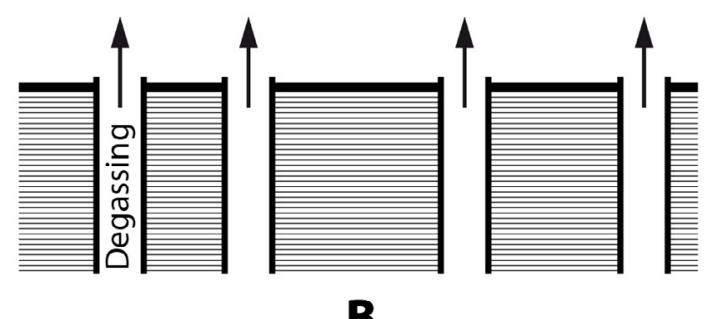

B
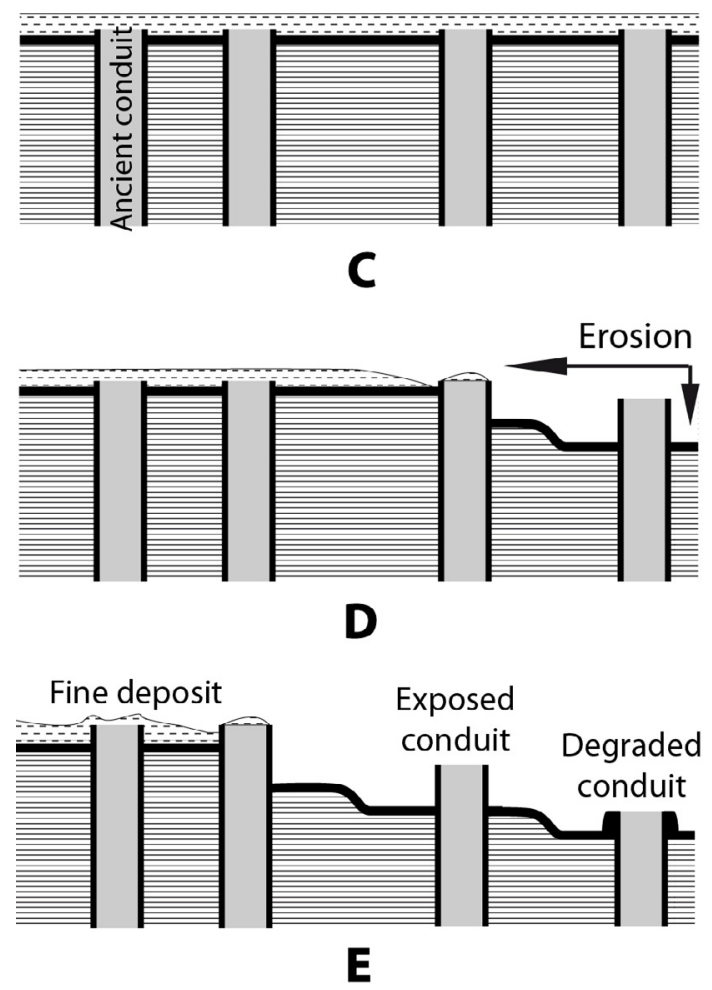

Fig. 19. Scenario for the formation and evolution of roundish features as ancient degassing conduits. A) Initial nucleus surface, covered by fine material. B) Formation of active degassing conduits, resulting in the erosion of the fine material. C) Activity of degassing conduits stops and fine material progressively covers them. D) Differential erosion starts on the surrounding terrains, revealing ancient degassing conduits (no longer active) from north to south. E) Current state: the ancient conduits are more or less degraded depending on how long they were exposed to solar illumination. Fine material still covers or hides the less exposed conduits.

\subsection{Boulders}

Boulders on Imhotep are mostly related to mass wasting. The mass wasting of the northern and western part of Imhotep are clearly associated with the surrounding scarps, and more particularly so on the southward slopes (Fig. 15). The profusion of boulders in the east may be mass wasting from the eastern highlands, but the top of the mass wasting is flat, which makes this 


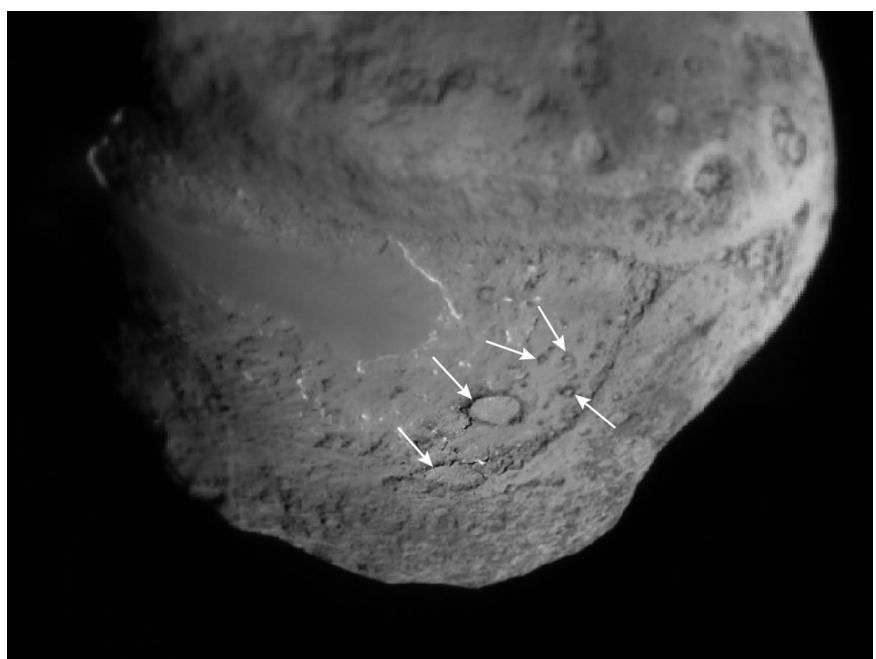

Fig. 20. Roundish features on comet 9P/Tempel 1, from tens of meters to $350 \mathrm{~m}$ for the largest ones.

hypothesis doubtful. An in situ conglomerate that is eroding and then reveals its constitutive boulders seems more likely.

As discussed in previous paragraphs, boulders likely are the erosion products of rocky terrains. But rocky terrains can be a homogeneous material or a conglomerate, so that boulders might also be remobilized boulders. We do not intend to answer the question of the primordial or evolutionary nature of boulders here, but it is clear that many boulders tend to be cut and recut by fractures.

The origin of the large isolated boulders in the middle of some smooth terrains is a key point (Fig. 2). With a size of tens of meters, they cannot be air-fall deposits (Sect. 4.1), unless a large, highly speculative outburst were assumed. We prefer the scenario where these boulders are the remnants of a previous mass wasting at the foot of the previous scarp location, when basins were less wide. They have slightly sunk since, as material accumulated around them. They do not show a dust deposit on their top, which further reinforces the idea that air-fall deposits only account for a small fraction of the smooth terrains.

\section{Conclusions}

We have shown that the Imhotep region is of double interest: its location near the equator, which makes it representative of a region illuminated from aphelion to perihelion that is even potentially active at perihelion, and its wide variety of morphologies, which allows examining a broad panel of processes. Although it is very low, gravity plays a significant role in the formation and evolution of the regional geomorphology, implying mass wasting and transport of materials. Cometary processes are responsible for the formation of roundish cylindrical features by gas pressure and contribute to the erosion of the cliffs by sublimation, with the highest intensity expected at perihelion.

This overview of the geomorphology of Imhotep and of the processes responsible for its landscape allow us to propose a scenario for the formation and evolution of this region:

\section{Cyclic and on-going processes:}

1. Formation of basins - the collapse of large cavities, tens of meters or more, leads to the formation of basins. These cavities are primordial voids, resulting from the nucleus formation process. Some first-generation basins may no longer be visible today.
2. Enlargement and infill of basins - the sublimation of ices leads to the erosion of basins and to their progressive infill with fine material and boulders by mass wasting.

3. Formation of smooth terrains - the degradation of boulders and fine material from mass wasting, plus air-fall deposits, leads to the accumulation of smooth material in gravitational lows. Fracturing probably exacerbates this degradation process.

\section{Transient events:}

1. Formation of basin $\mathrm{F}$ - the formation of this large structure and associated fractures was triggered by either an impact or the rising up of a gas bubble from the interior of the nucleus.

2. Formation of roundish features - these cylindrical features are probably ancient degassing conduits revealed by the differential erosion of surrounding less compacted materials.

The layers suggested by the terraces around basin F probably predate the formation of this basin. They might either be primordial, resulting from the nucleus formation process, or result from an ancient evolutionary process.

This scenario implies a general flattening of the region toward smooth terrains, a process similar to that observed on Earth, where old geological regions tend to be flatter than young regions. The next step to further constrain this scenario is to detect and monitor changes on the Imhotep region with Rosetta, as $67 \mathrm{P}$ approaches perihelion. A fundamental question is where erosion occurs today on Imhotep, if it does at all.

Acknowledgements. OSIRIS was built by a consortium of the Max-PlanckInstitut für Sonnensystemforschung, Göttingen, Germany, CISAS-University of Padova, Italy, the Laboratoire d'Astrophysique de Marseille, France, the Instituto de Astrofísica de Andalucia, CSIC, Granada, Spain, the Research and Scientific Support Department of the European Space Agency, Noordwijk, The Netherlands, the Instituto Nacional de Técnica Aeroespacial, Madrid, Spain, the Universidad Politéchnica de Madrid, Spain, the Department of Physics and Astronomy of Uppsala University, Sweden, and the Institut für Datentechnik und Kommunikationsnetze der Technischen Universität Braunschweig, Germany. The support of the national funding agencies of Germany (DLR), France (CNES), Italy (ASI), Spain (MEC), Sweden (SNSB), and the ESA Technical Directorate is gratefully acknowledged. We thank David Romeuf from the University Claude Bernard Lyon 1 (France) for creating the red and blue anaglyph in Fig. 9.

\section{References}

Abe, S., Mukai, T., Hirata, N., et al. 2006, Science, 312, 1344

A'Hearn, M. F., Belton, M. J. S., Delamere, W. A., et al. 2005, Science, 310, 258

Basilevsky, A., \& Keller, H. 2007, Sol. Syst. Res., 41, 109

Belton, M. J., \& Melosh, J. 2009, Icarus, 200, 280

Belton, M. J., Thomas, P., Veverka, J., et al. 2007, Icarus, 187, 332

Belton, M. J., Thomas, P., Carcich, B., et al. 2013, Icarus, 222, 477

Bockelée-Morvan, D., Crovisier, J., Mumma, M., \& Weaver, H. 2004, Comets II, 391

Boehnhardt, H. 2004, Comets II, 1, 301

Britt, D., Boice, D., Buratti, B., et al. 2004, Icarus, 167, 45

Brownlee, D. E., Horz, F., Newburn, R. L., et al. 2004, Science, 304, 1764

Capaccioni, F., Coradini, A., Filacchione, G., et al. 2015, Science, 347, http: // www. sciencemag.org/content/347/6220/aaa0628.full.pdf

Capaccioni, F., Filacchione, G., \& Erard, S. 2014, AGU Fall Meeting

Colwell, J. E., Gulbis, A. A., Horányi, M., \& Robertson, S. 2005, Icarus, 175, 159

Delbó, M., Libourel, G., Wilkerson, J., et al. 2014, Nature, 508, 233

Dombard, A. J., \& Freed, A. M. 2002, Geophys. Res. Lett., 29, 65

Dombard, A. J., Barnouin, O. S., Prockter, L. M., \& Thomas, P. C. 2010, Icarus, 210,713

Dones, L., Weissman, P. R., Levison, H. F., et al. 2004, Comets II, 1, 153

Duncan, M., Levison, H., \& Dones, L. 2004, Comets II, 1, 193

El-Maarry, M. R., Thomas, N., Giacomini, L., et al. 2015, A\&A, 583, A26 
Fornasier, S., Hasselmann, P. H., Barucci, M. A., et al. 2015, A\&A, 583, A30 Gaskell, R. W., Barnouin-jha, O. S., Scheeres, D. J., et al. 2008, Meteoritics Planet. Sci., 43, 1049

Groussin, O., \& Lamy, P. 2003, A\&A, 412, 879

Gulkis, S., Allen, M., von Allmen, P., et al. 2015, Science, 347, 709

Hartogh, P., Lis, D. C., Bockelée-Morvan, D., et al. 2011, Nature, 478, 218

Jorda, L., Gaskell, R., Hviid, S., \& Capanna, C. 2014, AGU Fall Meeting

Keller, H. U., Küppers, M., Fornasier, S., et al. 2007, Icarus, 191, 241

Keller, H. U., Mottola, S., Davidsson, B., et al. 2015, A\&A, 583, A34

Kelley, M. S., Lindler, D. J., Bodewits, D., et al. 2013, Icarus, 222, 634

Lee, P. 1996, Icarus, 124, 181

Love, S. G., Hörz, F., \& Brownlee, D. E. 1993, Icarus, 105, 216

Massironi, M., Simioni, E., Marzari, F., et al. 2015, Nature, accepted

Miyamoto, H., Yano, H., Scheeres, D. J., et al. 2007, Science, 316, 1011

Molaro, J., \& Byrne, S. 2012, J. Geophys. Res.: Planets, 117

Pajola, M., Vincent, J.-B., Güttler, C., et al. 2015, A\&A, 583, A37

Pochat, N., Vance, S., \& Collins, G. 2009, in AGU Fall Meeting Abstracts, 1, 1289

Pommerol, A., Thomas, N., El-Maarry, M. R., et al. 2015, A\&A, 583, A25 Richardson, J. E., Melosh, H. J., \& Greenberg, R. 2004, Science, 306, 1526

Richardson, J. E., Melosh, H. J., Greenberg, R. J., \& O'Brien, D. P. 2005, Icarus, 179,325

Sierks, H., Barbieri, C., Lamy, P. L., et al. 2015, Science, 347, 1044

Sunshine, J. M., A'Hearn, M. F., Groussin, O., et al. 2006, Science, 311, 1453

Tauber, F., \& Kuhrt, E. 1987, Icarus, 69, 83

Thomas, N., Davidsson, B., El-Maarry, M. R., et al. 2015a, A\&A, 583, A17

Thomas, N., Sierks, H., Barbieri, C., et al. 2015b, Science, 347, http://www. sciencemag. org/content/347/6220/aaa0440. full.pdf

Thomas, P. 1993, Icarus, 105, 326

Thomas, P., Joseph, J., Carcich, B., et al. 2002, Icarus, 155, 18

Thomas, P. C., Veverka, J., Belton, M. J., et al. 2007, Icarus, 187, 4

Thomas, P., A'Hearn, M., Belton, M., et al. 2013a, Icarus, 222, 453

Thomas, P., A'Hearn, M. F., Veverka, J., et al. 2013b, Icarus, 222, 550

Tubiana, C., Snodgrass, C., Bertini, I., et al. 2015, A\&A, 573, A62

Vanicek, P., \& Krakiwsky, E. J. 1986, Elsevier Science Pub. Co., 1, 697

Vincent, J., Lara, L., Tozzi, G., Lin, Z., \& Sierks, H. 2013, A\&A, 549, A121

Vincent, J.-B., Oklay, N., Marchi, S., Höfner, S., \& Sierks, H. 2014, Planet. Space Sci., 101, 186

Weidenschilling, S. 2004, Comets II, 745, 97

Weissman, P. R., Asphaug, E., \& Lowry, S. C. 2004, Comets II, 1, 337

Werner, R., \& Scheeres, D. 1996, Celest. Mech. Dyn. Astron., 65, 313

1 Aix-Marseille Université, CNRS, LAM (Laboratoire d'Astrophysique de Marseille) UMR 7326, 13388 Marseille, France

e-mail: anne-therese.auger@lam.fr

2 Laboratoire GEOPS (Géosciences Paris Sud), Bât. 509, Université Paris Sud, 91405 Orsay Cedex, France

3 Institut de Mécanique Céleste et de Calcul des Éphémérides, UMR 8028, 77 avenue Denfert Rochereau, 75014 Paris, France

${ }^{4}$ Planetary Science Institute, Tucson, AZ 85719, USA

5 Physikalisches Institut, Sidlerstr. 5, University of Bern, 3012 Bern, Switzerland
${ }^{6}$ Max-Planck-Institut für Sonnensystemforschung, 37077 Göttingen, Germany

7 Department of Physics and Astronomy, Padova University, Vicolo dell'Osservatorio 3, 35122 Padova, Italy

${ }^{8}$ Centro de Astrobiologia (INTA-CSIC), 28691 Villanueva de la Canada, Madrid, Spain

9 International Space Science Institute, Hallerstrasse 6, 3012 Bern, Switzerland

10 Scientific Support Office, European Space Agency, 2201 Noordwijk, The Netherlands

11 Department of Physics and Astronomy, Uppsala University, Box 516, 75120 Uppsala, Sweden

12 PAS Space Research Center, Bartycka 18A, 00716 Warszawa, Poland

${ }^{13}$ Institute for Geophysics and Extraterrestrial Physics, 38106 Braunschweig, Germany

14 Department of Astronomy, University of Maryland, College Park, MD 20742-2421, USA

15 LESIA, Obs. de Paris, CNRS, Univ Paris 06, Univ. Paris-Diderot, 5 place J. Janssen, 92195 Meudon, France

16 LATMOS, CNRS/UVSQ/IPSL, 11 boulevard d'Alembert, 78280 Guyancourt, France

17 University of Padova, CISAS, via Venezia 15, 35100 Padova, Italy

18 Department of Mech. Engineering University of Padova, via Venezia 1, 35131 Padova, Italy

19 CNR-IFN UOS Padova LUXOR, via Trasea 7, 35131 Padova, Italy

${ }^{20}$ UNITN, Universit di Trento, via Mesiano, 77, 38100 Trento, Italy

21 INAF-Osservatorio Astronomico, via Tiepolo 11, 34143 Trieste, Italy

22 Instituto de Astrofisica de Andalucía (CSIC), Glorieta de la Astronomía s/n, 18008 Granada, Spain

23 Institute of Planetary Research, DLR, Rutherfordstrasse 2, 12489 Berlin, Germany

24 Institute for Space Science, Nat. Central Univ., 300 Chung Da Rd., 32054 Chung-Li, Taiwan

25 Operations Department, European Space Astronomy Centre/ESA, PO Box 78, 28691 Villanueva de la Canada, Madrid, Spain

26 Southwest Research Institute, 1050 Walnut St., Boulder, CO 80302, USA

27 INAF, Osservatorio Astronomico di Padova, 35122 Padova, Italy

28 Centro di Ateneo di Studi ed Attivitá Spaziali "Giuseppe Colombo" (CISAS), University of Padova, 35131 Padova, Italy

29 Institut für Datentechnik und Kommunikationsnetze der TU Braunschweig, Hans-Sommer-Str. 66, 38106 Braunschweig, Germany

30 University of Padova, Department of Information Engineering, Via Gradenigo 6/B, 35131 Padova, Italy

31 Instituto Nacional de Tecnica Aeroespacial, Carretera de Ajalvir, p.k. 4, 28850 Torrejon de Ardoz, Madrid, Spain 